\title{
Using deep images and simulations to trace collisional debris around massive galaxies
}

\author{
Pierre-Alain Duc \\ AIM Paris-Saclay \\ Service d'astrophysique, CEA-Saclay, 91191 Gif sur Yvette, France \\ email: paduc@cea.fr
}

\begin{abstract}
Deep imaging programs, such as MATLAS which has just been completed at the CFHT, allows us to study with their diffuse light the outer stellar populations around large number of galaxies. We have carried out a systematic census of their fine structures, i.e. the collisional debris from past mergers. We have identified among them stellar streams from minor mergers, tidal tails from gas-rich major mergers, plumes from gas-poor major mergers, and shells from intermediate mass mergers. Having estimated the visibility and life time of each of these structures with numerical simulations, we can reconstruct the past mass assembly of the host galaxy. Preliminary statistical results based on a sample of 360 massive nearby galaxies are presented.
\end{abstract}

Keywords. galaxies: interactions

\section{Introduction}

Several teams are now actively involved in deep imaging programs of nearby galaxies. These surveys use different techniques and instruments: long exposures either on small, amateur-like, telescopes (e.g. Martínez-Delgado et al. 2010; Rich et al., this volume) or 10-meter class ones (e.g. Trujillo \& Fliri 2016; Tanaka et al., this volume), instruments dedicated to Low Surface Brightness (LSB) studies (e.g. "Dragonfly", van Dokkum et al. 2014), shallow surveys, stacking hundreds of galaxies (e.g. SDSS, DSouza et al. 2014), multi-visited fields initially used for calibration purposes (SDSS-Stripe82, Fliri \& Trujillo 2016), or LSB optimized observations on 2-4 meter class telescopes (among others, ESO/VST, Iodice et al. 2016; Blanco-CTIO/DECAM, Muñoz et al. 2015; CFHT/MegaCam, NGVS, Ferrarese et al. 2012). We have exploited the latter approach, and in particular MegaCam on the CFHT, as it offers a number of advantages: (a) a (local) surface brightness limit of 29 mag. $\operatorname{arcsec}^{-2}$ reached in only $45 \mathrm{~min}$, instead of 10-30 hours when using small telescopes. This could be achieved thanks to a dedicated observing strategy and pipeline that resulted in a gain of several mag with respect to traditional imaging surveys with the same total integration time (b) the ability to conduct, though large programs, complete volume limited surveys instead of in-depth studies of a few specific galaxies (c) a good sensitivity to extended LSB structures while benefiting from the excellent image quality of MegaCam and seeing conditions of the Mauna Kea site. This allows us to conduct additional studies such as the census of globular clusters, another tracer of the outskirts of galaxies.

The Large Program MATLAS, presented here, complemented by the NGVS which has mapped the whole Virgo cluster, has targeted 240 nearby massive Early Type galaxies (ETGs) from the Atlas3D sample (Cappellari et al. 2011; Duc et al. 2015). As a bonus, 120 Late Type galaxies (LTGs) located in the large MegaCam field of view, as well as hundreds of dwarf galaxies, benefit from deep images. 


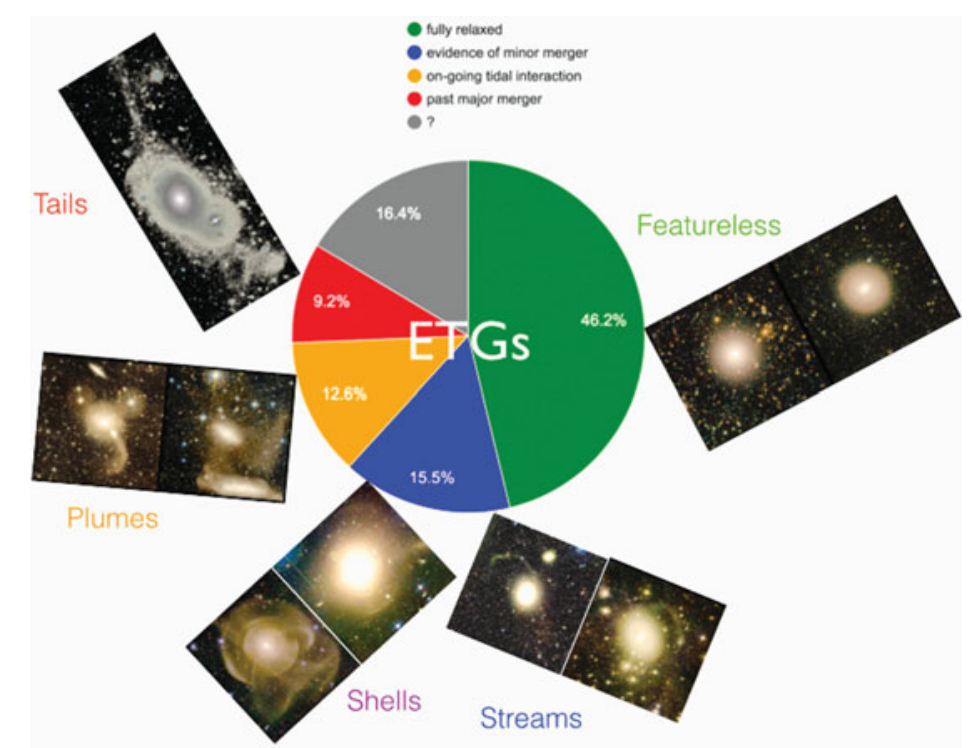

Figure 1. The classification scheme used for the MATLAS deep imaging survey, which is based on the identification of various types of collisional debris. Examples of tails, plumes, shells and streams are illustrated in the insets. The pie chart summarizes the preliminary statistical results for the population of 240 massive Early Type Galaxies.

\section{Census of collisional debris in observations and simulations}

Like galaxy archeology done in local galaxies with resolved stellar populations, the deep imaging programs exploiting the diffuse light focus on the external regions of galaxies. The individual collisional debris found there trace the last merging events, while the extended stellar halos have an older but less precise memory of the mass assembly. I present here our efforts to make the census of the fine structures around ETGs and LTGs, based on the CFHT multi-band deep images and the analysis of numerical simulations. The originality of our approach is to make a distinction between the various types of collisional debris, as each of them gives different pieces of information on the past accretion episodes.

As illustrated in Fig. 1, minor mergers produce narrow stellar streams; major wet mergers are characterized by prominent star-forming tidal tails; major dry mergers induce the formation of faint broad gas-poor plumes; shells trace back intermediate mass mergers while the absence of any structure may indicate cold gas accretion and a secular evolution. Streams, tails, plumes and shells were identified by eye by several team members, based on surface brightness and color maps, as well as residual images resulting from the subtraction of a model of the host galaxy.

Collisional debris are intrinsically faint and furthermore evaporate with time or may be destroyed by subsequent accretion events. Their detection depends on the type of structures, on their orientation on the sky, and above all on the achieved surface brightness limit. Numerical simulations are absolutely required to estimate their visibility time and thus reconstruct the past merging history of galaxies. This motivated us to analyse a set of simulations made in cosmological context. Snapshots were produced at different times, orientations on the sky, and surface brightness limits, and then mixed. Team members were invited to identify by eye and count the various types of fine structures. The evolution of the number of structures as a function of time was then reconstructed, and compared with the merging history of the galaxy, which is known by construction (Mancillas et al., in prep, see Fig. 2). 

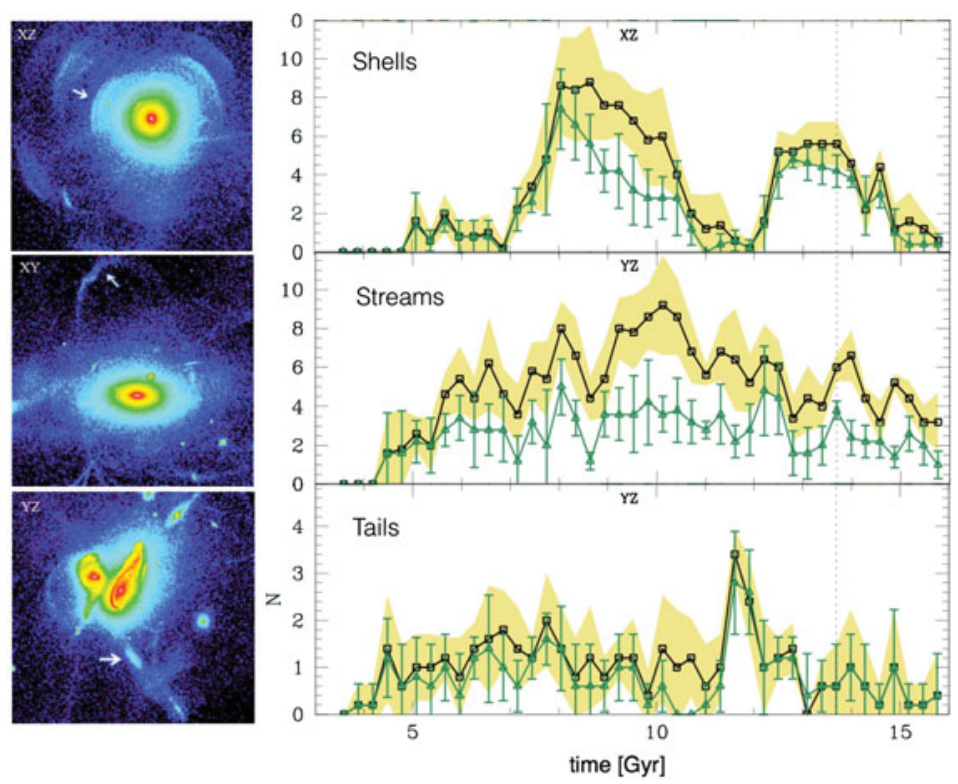

Figure 2. Simulation of the mass assembly of a massive galaxy traced by their fine structures. The evolution of the number of shells, streams and tails as a function of time is shown to the right. The green curve relies on the analysis of snapshots cut at the limiting surface brightness of the MATLAS observations. Examples of each of these structures are shown to the left. The images correspond to the orientation for which they are best seen.

\section{Preliminary results}

Thanks to the depth of the survey, we found - unsurprisingly - a fraction of tidally perturbed massive galaxies raising from about $15 \%$ in classical surveys (e.g. Atkinson et al. 2013) to $40 \%$ (see Fig. 1). Our large sample of 360 galaxies allows us to obtain statistically significant trends. In particular, ETGs with stellar masses above $10^{11} \mathrm{M}_{\odot}$, and, in particular, among them the slow rotators, i.e. the galaxies with a stellar kinematics showing no indication of rotation, seem to have experienced recent wet major mergers with a frequency increased by a factor of 3. While a significant fraction of LTGs in our sample show evidence for on-going tidal interactions, only few of them exhibit streams and tails indicative of a past strong minor / major merger activity. Preliminary results further indicate a mild dependence of the fraction of tidally perturbed galaxies on the large scale environment. All these trends on the fine structure fraction, once assessed with a more detailed analysis and completed by studies of the outer stellar halo, will be compared with predictions from cosmological simulations.

\section{References}

Atkinson, A., et al. 2013, ApJ, 765, 28

Cappellari, M., et al. 2011, MNRAS, 416, 1680

D'Souza, R., et al. 2014, MNRAS, 443, 1433

Duc, P.-A., et al. 2015, MNRAS, 446, 120

Ferrarese, L., et al. 2012, ApJS, 200, 4

Iodice, E., et al. 2016, ApJ, 820, 42

Martínez-Delgado, D., et al. 2010, AJ, 140, 962

Muñoz, R., et al. 2015, ApJL, 813, L15

Trujillo, I \& Fliri, K. 2016, ApJ, in press

van Dokkum, P., et al. 2014, ApJL, 782, L24 